\title{
Oral cavity manifestations by COVID-19 infections: a review
}

\author{
Santosh Kumar Swain ${ }^{1 *}$, Priyanka Debta ${ }^{2}$, Ansuman Sahu ${ }^{1}$, Smarita Lenka ${ }^{1}$
}

\begin{abstract}
${ }^{1}$ Department of Otorhinolaryngology and Head and Neck Surgery, IMS and SUM Hospital, Siksha “O” Anusandhan University, Bhubaneswar, Odisha, India

${ }^{2}$ Department of Oral Pathology and Microbiology, IDS and SUM Hospital, Siksha “O” Anusandhan University, Bhubaneswar, Odisha, India
\end{abstract}

Received: 30 May 2021

Accepted: 15 July 2021

\section{*Correspondence:}

Dr. Santosh Kumar Swain,

E-mail: santoshvoltaire@yahoo.co.in

Copyright: (C) the author(s), publisher and licensee Medip Academy. This is an open-access article distributed under the terms of the Creative Commons Attribution Non-Commercial License, which permits unrestricted non-commercial use, distribution, and reproduction in any medium, provided the original work is properly cited.

\begin{abstract}
Coronavirus disease 2019 (COVID-19) is a threat to the global health caused by severe acute respiratory syndrome coronavirus 2 (SARS-CoV-2). The lungs are the primary site of infection in COVID-19 patient and the symptoms ranges from mild flu like manifestations to fulminant pneumonia and respiratory failure. COVID-19 infection also significantly affects the oral cavity and salivary glands with oral mucosal manifestations. Other than airway manifestations, COVID-19 patients are presenting with oral cavity lesions such as aphthous like ulcers, glossitis, oral mucositis or stomatitis, oral candidiasis and herpetic recurrences. These oral lesions are often associated with immunocompromised patients and elderly age. Direct involvement of the SARS-CoV-2 virus for development of oral ulcers remains uncertain. The salivary gland related symptoms and taste disturbances are highly common in COVID19 patients. In COVID-19 patient, certain presentations like ulcers or blisters or diffuse reddish lesions affect both keratinized and non-keratinized tissues of the oral cavity. These lesions are found in palate, lip mucosa, buccal mucosa and tongue. The ulceration and blisters of the oral cavity are more often seen. There is still a gap of knowledge related to the oral manifestations of the COVID-19 infections and its impact on the oral cavity. This review article discussed the details of the oral cavity lesions in COVID-19 patients.
\end{abstract}

Keywords: COVID-19 patient, SARS-CoV-2, Oral cavity, Aphthous like lesions, Dysgeusia

\section{INTRODUCTION}

COVID-19 is highly infectious acute respiratory disease caused by SARS-CoV-2. ${ }^{1}$ COVID-19 patients present with wide variety of symptoms and signs. COVID-19 infections is usually present with acute onset and also require multiple treatment measures which can be associated with certain adverse effects in the oral cavity resulting few opportunistic infections like recurrent herpes simplex virus infection and oral ulcers because of the immunocompromised conditions and xerostomias related to the decreased salivary flow. ${ }^{2}$ At the beginning of the COVID-19 pandemic, it was though that absence of the oral mucosal involvement is an important differentiating feature of the COVID-19 exanthema relative to other viral causes of the exanthemas. Recently, the SARS-CoV-2 virus has been found from the saliva of the COVID-19 positive patient and it has been confirmed that reverse transcriptase polymerase chain reaction (RTPCR) from the saliva which can be more sensitive in comparison to the nasopharyngeal test. ${ }^{3}$ Furthermore, angiotensin converting enzyme (ACE-2) has been detected in the mucosal lining of the oral cavity, especially more densely on the dorsum of the tongue and salivary glands relative to the buccal mucosa and palates. ${ }^{4}$ Certain factors of the patients aggravate the development of the oral cavity lesions such as older age of the patient, severity of the COVID-19 infection, lack of the oral hygiene, stress, opportunistic infection, underlying 
diabetes mellitus, immunosuppressive medication, immunocompromised disease and trauma secondary to intubation. ${ }^{5}$ The management of the oral cavity lesions in COVID-19 patients include proper oral hygiene and treatment of the exact etiology of the oral lesions. This review article was aimed to describe details of the oral cavity lesions in the COVID-19 patient.

\section{METHODS OF LITERATURE SEARCH}

The research articles related to oral cavity manifestations by COVID-19 infections were searched through multiple approaches. First, we conducted an online search of the PubMed, Scopus, Google Scholar and Medline databases with word oral cavity manifestations, COVID-19 infections, gustatory dysfunction and candidiasis in COVID-19 patients. A search strategy using preferred reporting items for systemic reviews and meta-analysis guidelines was developed. Randomized controlled studies, observational studies, comparative studies, case series and case reports were evaluated for the eligibility. This paper focuses only on oral cavity lesions in COVID19 patients. Review articles with no primary research data were also excluded. The abstracts of the published articles were identified by this search method and other articles were identified manually from the citations. This review article reviewed the epidemiology, etiopathology of the oral lesions by SARS-CoV-2 virus infections, different oral cavity lesions and its management in COVID-19 patients. This review article presented a baseline from where further prospective trials can be designed and help as a spur for further research in this important clinical aspect as oral cavity lesions of the COVID-19 patients where not many studies were done.

\section{EPIDEMIOLOGY}

SARS-CoV-2 emerged in Wuhan, China in December 2019 and spread globally. 6 The COVID-19 pandemic quickly affected more than ten millions of people worldwide. $^{7}$ The outbreak of COVID-19 infection urges epidemiological and clinical researchers to document sensitive case definitions for tracking all true positive cases where there is emerging evidence of the oral cavity lesions. There are conflicting reports related to the prevalence of several symptoms and signs in the oral cavity of the COVID-19 patients. $^{8}$ There is lack of robust epidemiological studies regarding oral manifestations of the COVID-19 patients, which indicate that this clinical issue is neglected by patients and clinicians. The oral cavity of the COVID-19 patients should be evaluated in case of prolonged hospitalization or immunosuppressive status which may help to patient's recovery. The systemic viral infections like human immune deficiency virus have lesions in the oral cavity and the progression of this systemic viral infections is usually associated with ranges of the oral cavity manifestations. ${ }^{9}$ The lesions of the oral cavity were found to have diagnostic and prognostic values. One study showed that most common oral cavity lesions in non-COVID patient were coated tongue
$(51.4 \%)$, leukoplakia $(13.8 \%)$, traumatic oral ulcers in $9.2 \% .^{10}$ In one study on COVID-19 patients, the oral cavity lesions included aphthous like ulcers (36.5\%), erythema $(25.7 \%)$, lichen planus $(16.2 \%){ }^{11}$

The exact cause of oral lesions in COVID-19 patients is still not known, so seems to be multifactorial. ${ }^{12}$ The appearance of the oral lesions in COVID-19 patients may be associated with direct or indirect action of SARSCoV-2 over the mucosal lining of the oral cavity, hypersensitivity of the drugs taken at the time of the COVID-19 infection or due to lower immunity of the patient by the disease itself or prolonged hospitalization. SARS-CoV-2 virus infects the human cells through angiotensin converting enzyme 2 (ACE-2) receptors as ACE-2 act as primary host cell receptor for this virus. ${ }^{12}$ SARS-CoV-2 virus binds to ACE-2 with help of spike like protein on its surface whereas ACE-2 serves as a cellular portal for entry of the virus which result in COVID-19 infection. ${ }^{13}$ Therefore the organ which show high expression of ACE-2 can become target for SARSCoV-2 infection and these organs are lungs, salivary glands and tongue. ${ }^{14}$ The involvement of the salivary glands and tongue result in loss of taste sensation and ulcers in the oral cavity because of the destruction of the keratinocytes and oral fibroblasts. ${ }^{12}$ High viral loads in the salivary glands and nasal secretion can lead to oral changes in COVID-19 infection, which indicates the direct impact of the virus on the tissues of the oral cavity. ${ }^{15}$ The SARS-CoV-2 has also indirect influence on the immune system of the body, resulting into get opportunistic infections like recurrent herpes simplex (HSV-1) infections and ulcerations in the oral cavity. ${ }^{16}$ SARS-CoV-2 transmitted via two routes, either directly or indirectly. It is indirectly transmitted by saliva whereas it spread directly through sneezing, coughing and droplet inhalation or via direct contact with mucous membrane of the nasal cavity, oral cavity and ocular area. ${ }^{17}$

The oral cavity manifestations in COVID-19 patient may occur due to hypersensitivity reactions to drugs during the treatment of COVID-19 infection. In case of COVID19 infections, the cytokines storm and impaired or unregulated immune response may be associated with increased chance of the hypersensitive reactions in the oral cavity followed by ulcerative lesions. ${ }^{18}$ Moreover, the situations like stress and anxiety due to restrictions of the social life during COVID-19 pandemic may be the cause for development of aphthoid ulcers or herpetic recurrences. Thus, direct involvement of SARS-CoV-2 for development of the oral cavity lesions or ulcers remains uncertain. The oral ulcers associated COVID-19 patients may interfere with swallowing, chewing and speaking.

\section{ORAL CAVITY}

Oral cavity is described as a mirror reflecting the underlying health condition of the body. Proper examination of the oral cavity will give an early 
diagnosis and treatment as certain oral symptoms like oral ulcerations, gingival bleeding, dry mouth and discomfort in the oral cavity, halitosis, burning sensation or swallowing difficulty which are associated with certain systemic diseases. ${ }^{19}$ Oral transmission is considered as an important route for COVID-19 infection. ${ }^{20}$ Saliva also act as a source for diagnosis of the COVID-19 infection. ${ }^{21}$ So, it is important to maintain precautions during examining and performing any procedures in the oral cavity. There are different types of enanthema such as aphthous ulcers, petechiae, Koplik's spots, Nagayama's spot, papulovesicular or maculopapular lesions, white or red patches, swelling on gingival and lips have also been reported in different viral infections. ${ }^{21}$ The keratinized mucosal area like hard palate, gingiva and dorsum of the tongue and non-keratinized mucosal area like labial and buccal mucosa can be affected in COVID-19 infections. ${ }^{22}$ One study demonstrated approximately $7 \%$ of the COVID-19 patients presented with plaque like lesions on the dorsum of the tongue. ${ }^{23}$ Another study documented $29 \%$ of the COVID-19 cases shows enanthem. ${ }^{24}$ The oral cavity lesions in COVID-19 patient is found in both genders and not showing predilection to any specific male or female. The mean age of the affected patient is approximately 52 years of the age $( \pm 15$ years) and the clinical manifestations are quite heterogenous with varying types of lesions with its location. ${ }^{25}$ The most common type of the lesions in the oral cavity of COVID19 patient is ulceration in the mucosal lining. The oral cavity manifestations associated with COVID-19 infection have been documented like lingual pain in relation higher expression of ACE-2, the SARS-CoV-2 receptor, in epithelial cells of the tongue in comparison to other tissues of the oral cavity and also anosmia or ageusia induced by the inflammation in the oral cavity. ${ }^{26}$ The current literature showed that dysgeusia was only manifestation of the oral cavity.

\section{WHITE AND RED PLAQUES}

White and red patches or plaques are found on the dorsum of tongue, palate and gingiva of patients in confirmed or suspected COVID-19 patient. Candidiasis or oral thrush may occur due to long term antibiotic therapy, decreased immunity of the patient and decline of the oral hygiene can result in white patches (Figure 1). ${ }^{27}$ Oral and oropharyngeal candidiasis are found in COVID19 patients which often begins with colonization of the Candida species on the oral mucosa. Patients with oral candidiasis often present with local discomfort, taste disturbance, burning sensation in the oral cavity, glossodynia, dysphagia and aggravate the breathing difficulty. ${ }^{28}$

\section{APHTHOUS LIKE LESIONS}

Aphthous like lesions are found as multiple shallow ulcers with erythematous halo and yellowish-white pseudo membranes at the keratinized and non-keratinized mucosa of the oral cavity. Sometimes oral cavity lesions are seen simultaneously with systemic symptoms. Aphthous like lesions without presence of necrosis are found in younger patients in mild infections whereas aphthous like lesions with necrosis and hemorrhagic crusts seen more in elderly patients with lower immunity and severe infections. ${ }^{29}$ Regression of the lesions of the oral cavity is directly associated with improvement of the systemic diseases. Raised tumor necrosis factor (TNF) alpha in COVID-19 patients can result chemotaxis of the neutrophils in the oral mucosa and development of the aphthous like lesions. Stress and decreased immunity secondary to COVID-19 infection can cause such lesions in the oral cavity. ${ }^{30}$

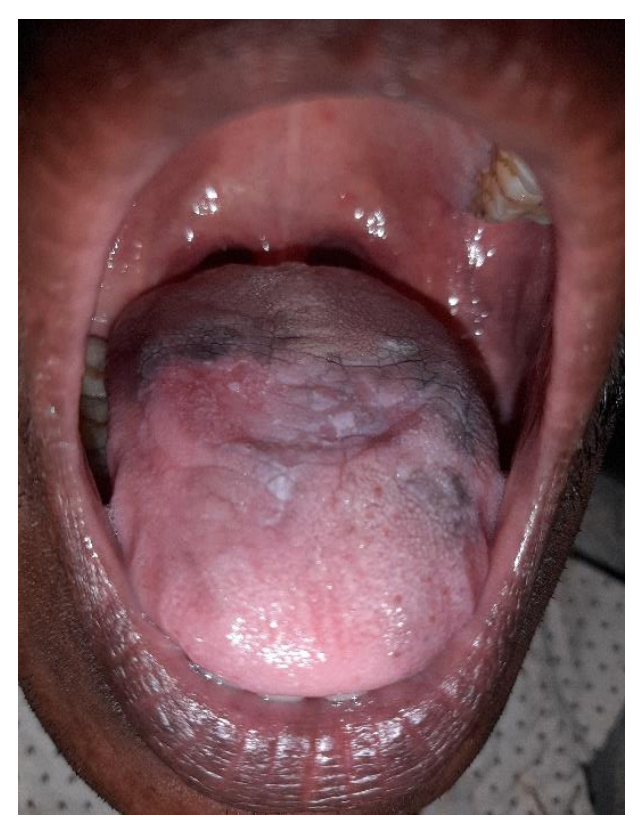

Figure 1: A COVID-19 patient presenting with oral thrush.

\section{XEROSTOMIA}

The COVID-19 infections often affect the salivary glands which reflected in different ways such as patient first complain pain or swelling in submandibular or submandibular glands and lead to dry mouth. These clinical findings reflect the protective role of the saliva which usually cleans the oral cavity constantly and the antiviral response against COVID-19. ${ }^{31}$ Hyposalivation is a potential complication of chronic sialadenitis caused by COVID-19 infection. Inflammation of the salivary glands result in fibrosis of the acinic and duct leading to hyposecretion of the salivary glands. Hyposalivation increases the chance of inorganic salt deposition on the ductal wall which induces the sialolithiasis which result in stenosis and dilation of the ducts. ${ }^{32}$ The incidence of the respiratory infection increases by enhancing adhesion of virus and colonization and destruction of the oral cavity mucosal linings and airways resulting in decreased antimicrobial peptides and proteins. ${ }^{33}$ 


\section{DYSGEUSIA}

Gustatory dysfunction is probably best known oral cavity manifestation of COVID-19 patient. ${ }^{34}$ (D1b) Dysgeusia is the first recognized oral cavity symptom of the COVID19 patient in approximately $38 \%$ of the patients, mostly in Europeans, American's females and patients with mild to moderate disease severity. ${ }^{35}$ Loss of taste sensation (ageusia) occurs in the viral infection of the olfactory cranial nerves or because of the rhinitis and nasal block. Ageusia is often associated with loss of smell (anosmia). Anosmia is associated with retro-nasal olfaction which is a sensory process, combining ortho-nasal smell and taste patterns and help to perceive the flavor. This mechanism is usually disturbed temporarily at the time of upper respiratory tract infection because of the mucosal inflammation and blockage of the nasal passage, so directly obstruct flavor and odor molecules from entering the olfactory cleft. ${ }^{36}$ Gustatory dysfunction or dysgeusia is a common oral cavity manifestation of COVID-19 patient. ${ }^{34,37}$ One study showed that salivary ducts are the first point of infection showing early presence of SARSCoV-2 in COVID-19 positive patient's saliva. ${ }^{32}$ This study hypothesized that SARS-CoV-2 result in acute or chronic sialadenitis which cause different diseases in the oral cavity. ${ }^{32}$ There are certain oral cavity diseases caused by directly COVID-19 infection. ${ }^{38}$

\section{HERPETIFORM LESIONS}

Herpetic form/zosteriform lesions are seen as multiple painful, yellowish-gray ulcers with erythematous surrounding on both keratinized and non-keratinized mucosa of the oral cavity. ${ }^{39}$ Geographic tongue may be found after recovery of herpetic lesions. Immunosuppression and stress are often associated with COVID-19 infection which result in appearance of the secondary type of herpetic gingivostomatitis. ${ }^{29}$ In some cases, COVID-19 positive patients present with blisters in the lip mucosa, desquamative gingivitis and skin rashes. Some may present painful inflammation of the tongue papillae which may lead to erythematous macule and ulcers. $^{40}$

\section{KAWASAKI LIKE DISEASE}

The oral lesions such as glossitis, cheilitis, erythematous and swollen tongue (described as strawberry tongue) are seen in COVID-19 patients with Kawasaki like disease (Kawa-COVID). ${ }^{12}$ The long period latency between beginning of the systemic symptoms such as respiratory or gastrointestinal) and onset of the oral or skin manifestations by delayed hyperactivation response of the immune symptoms. The secondary release of the acute inflammatory cytokines in respect to direct effects of the virus on the skin and oral mucosa result in release of the acute inflammatory mediators rather than direct impact of the viruses on the skin and oral mucosa. ${ }^{12}$

\section{MELKERSSON-ROSENTHAL SYNDROME}

In Melkersson-Rosenthal syndrome, patients usually complain of fissured tongue, swelling of lips and facial nerve paralysis. Patients with features of MelkerssonRosenthal syndrome with increased level of C-reactive protein (CRP) and ground glass appearance in computed tomography (CT) scan of the chest confirm the COVID19. ${ }^{41}$ This patient is cured completely by treatment of the COVID-19 infection. ${ }^{41}$

\section{ANGINA BULLOSA LIKE LESIONS}

COVID-19 patients sometimes present with asymptomatic erythematous purple blisters without any evidence of spontaneous bleeding on the tongue and hard palate. $^{42}$

\section{PETECHIAE}

In COVID-19 patients, petechiae were found on the palate, lower lip and oropharynx. The latency period for patients of petechiae is shorter in comparison to the patients with both petechiae and macular lesions. Thrombocytopenia in COVID-19 infection or certain drugs prescribed are the cause for petechiae. ${ }^{43}$

\section{VESICLES AND PUSTULES}

A report of COVID-19 positive pediatric patient showed fever, weakness, pain abdomen and diarrhea along with oral and acral erythematous popular exanthema. The oral lesions in this case showed vesicular eruptions and erosions on the buccal mucosa and tongue and these lesions were cured by one week. ${ }^{44}$ There was an adult patient presented with fever, dry cough, fatigue, dysgeusia, anosmia and positive for SARS-CoV-2 infection. This patient presented with erythema on hard palate and oropharynx with petechiae and pustules on the soft palate border. The diagnosis was suggested as enanthema due to COVID-19 and these lesions cured after few days. ${ }^{45}$

\section{ORAL MUCOSITIS}

There are non-specific lesions found in COVID-19 positive patient and the lesions may include erythematous-violaceous macules, papules, patches and plaques on the lip mucosa, tongue, hard palate and oropharynx. Thrombotic vasculopathy, vasculitis and hypersensitivity associated with COVID-19 patient may cause mucositis (Figure 2). Thrombotic vasculopathy, mucosal hypersensitivity secondary to COVID-19 infection and vasculitis are possible causes for mucositis in COVID-19 patient. $^{25}$ 


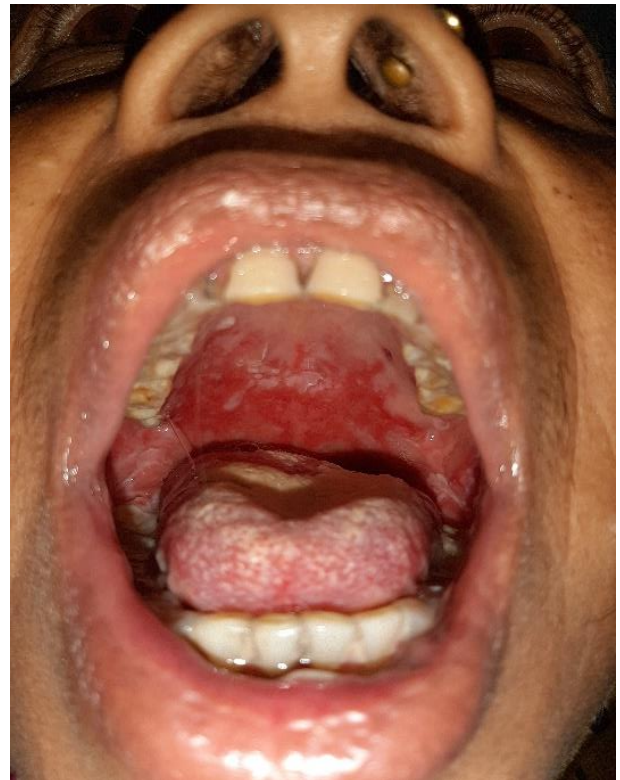

Figure 2: A COVID-19 patient presenting severe oral cavity mucositis.

\section{NECROTIZING PERIODONTAL DISEASE}

COVID-19 patient may present with fever, halitosis, submandibular gland lymphadenopathy and oral lesions. The oral lesions included diffuse erythematous lesions, painful edematous gingiva and necrosis of inter-papillary areas. The clinical diagnosis was necrotizing periodontal disease because of bacterial infection along with COVID19 infection. These oral lesions recovered usually after 5 to 7 days. ${ }^{46}$

\section{ULCER AND EROSIONS}

There are painful ulcerative or erosive lesions seen as irregular borders over the tongue, hard palate and labial mucosa in COVID-19 positive patient. Drug eruptions, vasculitis or thrombotic vasculopathy are thought to be the cause secondary to COVID-19 infection which result in ulcerative and erosive lesions. ${ }^{47}$

\section{ORAL MUCOSAL POST-INFLAMMATORY PIGMENTATION}

There was case report of pigmentation at the interpapillary gingiva. Increased inflammatory cytokines (interleukin-1, tumor necrosis factor) and arachidic acid metabolites (prostaglandins) secondary to stem cell factor production and basic-fibroblast growth factor from the keratinocytes of the basal layer result in postinflammatory pigmentation in the oral cavity of COVID19 patient. ${ }^{48}$

\section{TREATMENT}

Systemic or topical corticosteroid are helpful for treating the oral lesions of the COVID-19 patients. ${ }^{49}$
Chlorohexidine mouth wash or tetracycline can be prescribed for preventing the secondary infection in the oral cavity. ${ }^{50}$ The oral lesions often reduced within mean time of 7 days regardless of the treatment. ${ }^{50}$ Current studies suggest that the lesions of the oral cavity may be atypical manifestations in COVID-19 patients. ${ }^{50}$ Knowledge about these lesions in the oral cavity may help in early detection of the patients with asymptomatic disease and may aid in stopping the spread of the virus. However, the clinicians like dentist or otolaryngologists should follow strict precautionary such as wear personal protective equipment during examining the oral cavity of COVID-19 patients. ${ }^{47,51}$ The hypersensitivity reaction in the oral cavity or vaccination for COVID-19 infection may be the cause for oral cavity lesions, which should be carefully taken into consideration for managing such lesions. The drug which causes oral cavity manifestation in COVID-19 patients should be stopped. The adverse effects by vaccine for COVID-19 particularly in the oral cavity should be treated immediately. The oral cavity lesions in COVID-19 patient may occur by stress which should be consulted and managed by treatment of psychiatrist. Insufficient hygiene of the oral cavity in COVID-19 patient need local antiseptics such as hydrogen peroxide-based suspension for decreasing the viral burden. ${ }^{52}$

\section{CONCLUSION}

There are several lesions found in the oral cavity of the COVID-19 patient and the lesions include aphthous like ulcers, herpetiform lesions, candidiasis and Kawasaki disease like lesions. Impaired immunity and older age are important factors for predicting the lesions in the oral cavity of the COVID-19 patients. Lack of oral hygiene, stress, opportunistic infection underlying diseases like diabetes mellitus for manifesting oral lesions in COVID19 patients. Clinicians should keep these oral cavity lesions in mind during examination of the COVID-19 patients. Proper management of the COVID-19 infections and maintaining oral hygiene will help to improve the oral cavity lesions. The co-morbidities of the COVID-19 patients such as old age, diabetes mellitus, hypertension and stress are strongly associated with poor prognosis which trigger the lesions in the oral cavity. Stronger evidence is needed in the form of further studies with help of vitro molecular, in vivo and patient based observational studies to define the exact nature and underlying mechanisms of the oral cavity lesions in COVID-19 patient.

Funding: No funding sources Conflict of interest: None declared

Ethical approval: Not required

\section{REFERENCES}

1. Swain SK, Jena PP. Clinical implications and future perspective of COVID-19 pandemic-a review. Int J Adv Med. 2021;8(2):334-40. 
2. Dziedzic A, Wojtyczka R. The impact of coronavirus infectious disease 19 (COVID-19) on oral health. Oral Dis. 2021;27:703-6.

3. Swain SK, Behera IC. Performing laryngotracheobronchial procedure in coronavirus disease 2019 pandemic: a highly aerosol generating event in clinical practice. Matrix Science Medica. 2021;5(1):1-6.

4. Swain SK, Acharya S, Sahajan N. Otorhinolaryngological manifestations in COVID19 infections: an early indicator for isolating the positive cases. J Sci Soc. 2020;47(2):63-8.

5. Brandão TB, Gueiros LA, Melo TS, Prado-Ribeiro AC, Nesrallah AC, Prado GV, et al. Oral lesions in patients with SARS-CoV-2 infection: could the oral cavity be a target organ? Oral Surg Oral Med Oral Pathol Oral Radiol. 2021;131(2):45-51.

6. Swain SK, Pani SR. Incidence of hearing loss in COVID-19 patients: a COVID hospital-based study in the eastern part of India. Int. J Curr Res Rev. 2021;13:103-7.

7. Haynes BF, Corey L, Fernandes P, Gilbert PB, Hotez PJ, Rao S, et al. Prospects for a safe COVID19 vaccine. Sci Transl Med. 2020;12(568):948.

8. Swain SK, Kumar S. Infection control measures during COVID-19 pandemic-an otorhinolaryngological and head-and-neck perspective. Indian J Health Sci Biomed Res. 2021;14(1):3-11.

9. Donoso-Hofer F. Oral lesions associated with human immunodeficiency virus disease in adult patients, a clinical perspective. Rev Chilena Infectol. 2016;33(1):27-35.

10. Shet R, Shetty SRMK, Kumar MN, Yadav RD, Soumya S. A study to evaluate the frequency and association of various mucosal conditions among geriatric patients. J Contem Dent Pract. 2013;14(5):904-10.

11. Fidan V, Koyuncu H, Akin O. Oral lesions in COVID-19 positive patients. Am J Otolaryngol. 2021;42(3):102905.

12. Chiu JS, Lahoud-Rahme M, Schaffer D, Cohen A, Samuels-Kalow M. Kawasaki disease features and myocarditis in a patient with COVID19. Pediatr Cardiol. 2020;41(7):1526-8.

13. Ciaglia E, Vecchione C, Puca AA. COVID-19 infection and circulating ACE2 levels: protective role in women and children. Frontiers Pediatr. 2020;8:206.

14. Swain SK, Agrawala R. Mastoid surgery: a highrisk aerosol generating surgical procedure in COVID-19 pandemic. Int J Otorhinolaryngol Head Neck Surg. 2020;6(10):1941-6.

15. Tapia ROC, Labrador AJP, Guimaraes DM, Valdez LHM. Oral mucosal lesions in patients with SARSCoV-2 infection. Report of four cases. Are they a true sign of COVID-19 disease? Spec Care Dentist. 2020;40(6):555-560.

16. Swain SK, Choudhury J, Acharya S. Herpes zoster oticus among pediatric patients: Our experiences at a tertiary care teaching hospital. Indian Journal of Health Sciences and Biomedical Research (KLEU). 2020;13(3):215-20.

17. Peng X, Xu X, Li Y, Cheng L, Zhou X, Ren B. Transmission routes of 2019-nCoV and controls in dental practice. Int J Oral Sci. 2020;12(1):1-6.

18. Sakaida $\mathrm{T}$, Isao $\mathrm{T}$, Matsubara $\mathrm{A}$, Nakamura $\mathrm{M}$, Morita A. Unique skin manifestations of COVID19: is drug eruption specific to COVID-19? J Dermatol Sci. 2020;99(1):62-4.

19. Chi AC, Neville BW, Krayer JW, Gonsalves WC. Oral manifestations of systemic disease. Am Fam Physic. 2010;82(11):1381-8.

20. Hindson J. COVID-19: Faecal-oral transmission? Nature Rev Gastroenterol Hepatol. 2020;17(5):259.

21. Fernandes LL, Pacheco VB, Borges L, Athwal HK, Eduardo FDP, Bezinelli L, et al. Saliva in the diagnosis of COVID-19: a review and new research directions. J Dent Res. 2020;99(13):1435-43.

22. Rocha BAR, Souto GR, Grossmann SD, Aguiar MCD, de Andrade BA, Romañach MJ, et al. Viral enanthema in oral mucosa: a possible diagnostic challenge in the COVID-19 pandemic. Oral Diseases. 2021;27:776-8.

23. Biadsee A, Biadsee A, Kassem F, Dagan O, Masarwa S, Ormianer Z. Olfactory and oral manifestations of COVID-19: sex-related symptoms-a potential pathway to early diagnosis. Otolaryngol Head Neck Surg. 2020:163(4):722-8.

24. Jimenez-Cauhe J, Ortega-Quijano D, Perosanz-Lobo D, Burgos-Blasco P, Vañó-Galván S, FernandezGuarino M, et al. Enanthem in patients with COVID-19 and skin rash. JAMA Dermatol. 2020;156(10):1134-6.

25. Soares CD, Carvalho RA, Carvalho KA, Carvalho MG, Almeida OP. Oral lesions in a patient with COVID-19. Med Oral Patol Oral Cir Bucal. 2020;25(4):563-4.

26. Xu H, Zhong L, Deng J, Peng J, Dan H, Zeng X, et al. High expression of ACE2 receptor of 2019$\mathrm{nCoV}$ on the epithelial cells of oral mucosa. Int $\mathrm{J}$ Oral Sci. 2020;12:1-5.

27. Putra BE, Adiarto S, Dewayanti SR, Juzar DA. Viral exanthem with "pin and needles sensation" on extremities of COVID-19 patient. Int J Infect Dis. 2020;96:355-8.

28. Laudenbach JM, Epstein JB. Treatment strategies for oropharyngeal candidiasis. Expert Opin Pharmacother. 2009;10(9):1413-21.

29. Santos JAD, Normando AG, Silva RLD, Paula RMD, Cembranel AC, Santos-Silva AR, et al. Oral mucosal lesions in a COVID-19 patient: New signs or secondary manifestations? Int $\mathrm{J}$ Infect Dis. 2020;97:326-8.

30. Farshidfar N, Hamedani S. Hyposalivation as a potential risk for SARS-CoV-2 infection: inhibitory role of saliva. Oral Dis. 2021;27(3):750-1.

31. Swain SK, Das S, Padhy RN. Performing tracheostomy in intensive care unit-a challenge 
during COVID-19 pandemic. Siriraj Med J. 2020;72(5):436-42.

32. Han P, Ivanovski S. Saliva-friend and foe in the COVID-19 outbreak. Diagnostics. 2020;10(5):290.

33. Wang L, Shen Y, Li M, Chuang H, Ye Y, Zhao H, et al. Clinical manifestations and evidence of neurological involvement in 2019 novel coronavirus SARS-CoV-2: a systematic review and metaanalysis. J Neurol. 2020;267(10):2777-89.

34. Seirafianpour F, Sodagar S, Mohammad AP, Panahi P, Mozafarpoor S, Almasi S, et al. Cutaneous manifestations and considerations in COVID-19 pandemic: a systematic review. Dermatolog Ther. 2020;33(6):13986.

35. Melley LE, Bress E, Polan E. Hypogeusia as the initial presenting symptom of COVID-19. BMJ Case Rep. 2020;13(5):236080.

36. Swain SK. Smell and taste abnormalities: early clinical biomarkers for isolating the positive cases in COVID-19 pandemic. Int J Otorhinolaryngol Head Neck Surg. 2021;7(1):196-201.

37. Wang C, Wu H, Ding X, Ji H, Jiao P, Song H, et al. Does infection of 2019 novel coronavirus cause acute and/or chronic sialadenitis? Med Hypotheses. 2020;140:109789.

38. Carreras-Presas CM, Sánchez JA, López-Sánchez AF, Jané-Salas E, Pérez MLS. Oral vesiculobullous lesions associated with SARS-CoV-2 infection. Oral Dis. 2021;27:710-2.

39. Swain SK, Sahu MC, Behera IC. Management of Ramsay Hunt syndrome among HIV patients: Our experience in a tertiary care hospital of eastern India. Polish Ann Med. 2016;23(2):92-6.

40. Taşlidere B, Mehmetaj L, Özcan AB, Gülen B, Taşlidere N. Melkersson-Rosenthal syndrome induced by COVID-19. Am J Emerg Med. 2021;41:262-5.

41. Zou X, Chen K, Zou J, Han P, Hao J, Han Z. Singlecell RNA-seq data analysis on the receptor ACE2 expression reveals the potential risk of different human organs vulnerable to 2019-nCoV infection. Frontiers Med. 2020;14(2):185-92.

42. Aghazadeh N, Homayouni M, Sartori-Valinotti JC. Oral vesicles and acral erythema: report of a cutaneous manifestation of COVID-19. Int J Dermatol. 2020;59(9):1153-4.

43. Swain SK, Sahu A. Performing tracheostomy on COVID-19 pediatric patients at intensive care unit: Our experiences. Indian Journal of Health Sciences and Biomedical Research (KLEU). 2021;14(1):1316.

44. Kahraman FC, Kurlu HC. Mucosal involvement in a COVID19-positive patient: a case report. Dermatol Ther. 2009;14(6):272-7.

45. Aghazadeh N, Homayouni M, Sartori-Valinotti JC. Oral vesicles and acral erythema: report of a cutaneous manifestation of COVID-19. Int $\mathbf{J}$ Dermatol. 2020;59(9):1153-4.

46. Patel J, Woolley J. Necrotizing periodontal disease: Oral manifestation of COVID-19. Oral Dis. 2021;27:768-9.

47. Singh C, Tay J, Shoqirat N. Skin and mucosal damage in patients diagnosed with COVID-19: a case report. J Wound Ostomy Continence Nurs. 2020;47(5):435-8.

48. Corchuelo J, Ulloa FC. Oral manifestations in a patient with a history of asymptomatic COVID-19: case report. Int J Infect Dis. 2020;100:154-7.

49. Swain SK, Das S, Lenka S. Sudden Sensorineural Hearing Loss among COVID-19 Patients-Our Experiences at an Indian Teaching Hospital. Siriraj Medical Journal. 2021;73(2):77-83.

50. Swain SK, Behera IC. Managing pediatric otorhinolaryngology patients in coronavirus disease19 pandemic-a real challenge to the clinicians. Indian J Child Health. 2020;7(9):357-62.

51. Hasturk H, Nunn M, Warbington M. Efficacy of a fluoridated hydrogen peroxide-based mouthrinse for the treatment of gingivitis: a randomized clinical trial. J Periodontol. 2004;75(1):57-65.

Cite this article as: Swain SK, Debta P, Sahu A, Lenka S. Oral cavity manifestations by COVID-19 infections: a review. Int J Otorhinolaryngol Head Neck Surg 2021;7:1391-7. 